\title{
Noise Characteristics in High Precision GPS Positioning
}

\author{
A.R. Amiri-Simkooei, C.C.J.M. Tiberius, P.J.G. Teunissen, \\ Delft Institute of Earth Observation and Space systems (DEOS), Delft University of Technology, \\ Kluyverweg 1, 2629 HS Delft, The Netherlands, e-mail: A.AmiriSimkooei@TUDelft.nl
}

\begin{abstract}
In this contribution we present the results of three different studies in which the method of least-squares variance component estimation (LS-VCE) was used to infer the stochastic properties of GPS data. The three studies cover the GPS geometry-free model, the GPS coordinate time series model, and the GPS zero-baseline model. In the GPS geometry-free model, LS-VCE is applied to assess the precision of different observation types, correlation between observation types on L1 and L2, and satellite elevation dependence of the GPS observables precision. We show, for example, that the precision of code observations (for zero baseline) ranges from 10 to $15 \mathrm{~cm}$ depending on the satellite elevation and the type of the receiver used. The LSVCE time series analysis pertains to data of various permanent GPS tracking stations. It reveals that the noise can be divided into two components, namely white noise and flicker noise. We show that both noise components are spatially correlated (e.g. a correlation coefficient of about 0.8 over short distances between permanent stations). Finally, in the (classical) zero-baseline model, nonlinear LS-VCE is applied to assess the noise characteristics of GPS receivers based on covariance functions.
\end{abstract}

Keywords. Variance component estimation, noise characteristics, GPS time series, covariance function

\section{Introduction}

A proper choice of the data weight matrix is of importance for many parameter estimation problems. This also holds true for the many applications of GPS. A realistic description of the GPS data noise characteristics is required to obtain minimum variance estimators through the functional model. In the case of GPS, the functional model is welldeveloped and well documented. The same can not yet be said of the covariance matrix of the GPS data.
A systematic study of the GPS stochastic model is of course far from trivial. In this contribution we demonstrate this using three GPS applications.

Least-squares variance component estimation (LS-VCE) is employed to assess the noise characteristics of GPS data. Consider the following linear model of observation equations with a $p$-number of unknown (co)variance components

$$
\mathrm{E}\{\underline{y}\}=A x, \quad \mathrm{D}\{\underline{y}\}=Q_{y}=\sum_{k=1}^{p} \sigma_{k} Q_{k},
$$

where $y$ is the $m$-vector of observables, $x$ is the $n$-vector of parameters of interest, and $A$ is the $m \times n$ design matrix. The covariance matrix $Q_{y}$ is expressed as an unknown linear combination of the known $m \times m$ cofactor matrices $Q_{k}$ 's. The LS estimator for the $p$-vector of unknown (co)variance components $\sigma=\left[\begin{array}{llll}\sigma_{1} & \sigma_{2} & \ldots & \sigma_{p}\end{array}\right]^{T}$ can then be obtained as follows (Teunissen, 1988, Teunissen and AmiriSimkooei, 2007): $\underline{\hat{\sigma}}=N^{-1} \underline{l}$ with the $p \times p$ normal matrix $N$ and the $p$-vector $\underline{l}$ as

$$
n_{k l}=\frac{1}{2} \operatorname{tr}\left(Q_{y}^{-1} P_{A}^{\perp} Q_{k} Q_{y}^{-1} P_{A}^{\perp} Q_{l}\right),
$$

and

$$
\underline{l}_{k}=\frac{1}{2} \underline{y}^{T} Q_{y}^{-1} P_{A}^{\perp} Q_{k} Q_{y}^{-1} P_{A}^{\perp} \underline{y} ; k, l=1, \ldots, p,
$$

where the orthogonal projector is given as $P_{A}^{\perp}=$ $I-A\left(A^{T} Q_{y}^{-1} A\right)^{-1} A^{T} Q_{y}^{-1}$. The estimators obtained by this method are unbiased and of minimum variance. To apply the method, one starts with an initial guess for the (co)variance components and performs iterations. The iterative procedure is repeated until the estimated (co)variance components do not change with further iterations. Since the method is based on the least-squares principle, the inverse of the normal matrix $N$ automatically gives the covariance matrix of the estimated (co)variance 
components, namely $Q_{\hat{\sigma}}=N^{-1}$ which offers us measures of precision for the estimators.

\section{GPS Geometry-Free Model}

The GPS geometry-free observation model (GFOM) is one of the simplest approaches to processing and analysing data from a baseline and to integer GPS double differenced (DD) ambiguity estimation in particular. One advantage of using this model is its ease which stems from the linearity of the observation model and its independence of satellite orbit information. This model will be used in this section as a favorable model for estimation of (co)variance components via LS-VCE. The GFOM consists of two parts: the functional model and the stochastic model. The functional model relates the observations to the parameters of interest whereas the stochastic model describes the precision of and the mutual correlation between the observations. The functional model is based on the non-linearized DD dual frequency pseudo range and carrier phase observation equations. We use the LAMBDA method (see e.g. Teunissen (1993)) to fix the DD ambiguities and then introduce them into the model. For more information we refer to Jonkman (1998).

We need to come up with a realistic and adequate covariance matrix of the GPS observables in the case of the linear and simple GFOM. To this end we apply the LS-VCE. The construction of the covariance matrix (for undifferenced observables) starts from a scaled unit matrix per observation type and takes place in different steps. In this application, the following three characteristics of GPS observables will be evaluated: (1) the precision of the phase and code observations, (2) correlation between the observables on L1 and L2 frequencies, (3) satellite elevation dependence of the observables precision. Noise characteristics of the GPS observables have recently been assessed by different authors. We refer to e.g. Bischoff et al. $(2005,2006)$.

A data set was obtained from the Delfland 99 campaign in the Netherlands; one hour of Trimble 4000SSI zero baseline data, 8 satellites with four observation types, namely C1-P1-L1-L2, and a $1 \mathrm{sec}$ interval. In the sequel, the estimated (co)variance components for this receiver, over 3600 epochs divided into 360 10-epoch groups, will be presented.

The goal now is to estimate one variance component for each observation type when considering 10 epochs of all observations. We neglect here the satellite elevation dependence of the observables precision, the time correlation, the covariance between channels, and the covariance between different observation types. The final (co)variances can be obtained by multiplying the estimated (co)variance components with their a-priori values $30^{2} \mathrm{~cm}^{2}$ and $3^{2} \mathrm{~mm}^{2}$ for undifferenced code and phase observables, respectively. Note that the estimation of two individual variance components for L1 and L2 carrier phase data would cause the VCE problem to be ill-posed. The ill-posedness will be removed if we estimate one single variance component instead.

Figure 1 shows the groupwise estimates of variance components in the last iteration, using the full hour of the data for the L1/L2 phase and C1 and P2 code observations of Trimble receiver. The estimated factors, if multiplied with their initial values given in the cofactor matrices, give the final estimates. In Figure 1 the estimated variance components grow towards the end of the graph. It is likely because of one satellite, which is setting and has the lowest elevation angle (nearly $10^{\circ}$ ). Table 1 gives the standard deviation estimates (square-root of variance components) as well as their precision (in terms of undifferenced observables). The results indicate that the noise of GPS observations is about $0.3 \mathrm{~mm}$, $10 \mathrm{~cm}$, and $16 \mathrm{~cm}$ for phase, $\mathrm{C} 1$, and $\mathrm{P} 2$, respectively. The precision of the estimates are at micrometre and millimetre level for phase and code observations, respectively.

In addition to the variances, one can for instance estimate the covariance between $\mathrm{C} 1$ and $\mathrm{P} 2$. The satellite elevation dependence of the observables precision, the time correlation and the covariance between channels are disregarded. Figure 2 shows the groupwise estimates of the correlation

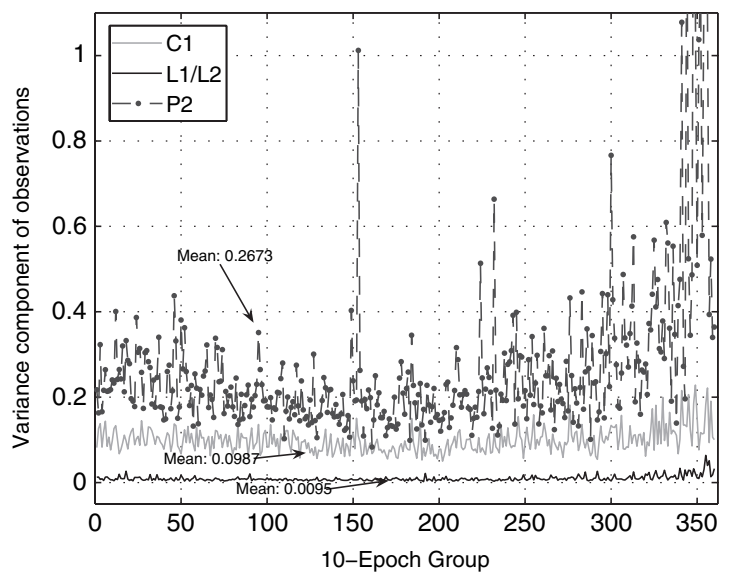

Fig. 1. Variance components groupwise estimated for L1 and L2 phase and $\mathrm{C} 1$ and $\mathrm{P} 2$ code observables using LS-VCE. Factors are to be multiplied by $30^{2} \mathrm{~cm}^{2}$ and $3^{2} \mathrm{~mm}^{2}$ for code and phase observables, respectively. 
Table 1. Standard deviation estimates of phase and code observables as well as their precision obtained using LS-VCE

\begin{tabular}{lll}
\hline Observation type & $\hat{\sigma}(\mathrm{mm})$ & $\sigma_{\hat{\sigma}}(\mathrm{mm})$ \\
\hline L1/L2 & 0.29 & 0.001 \\
C1 & 94.25 & 0.410 \\
P2 & 155.09 & 0.510 \\
\hline
\end{tabular}

coefficient. As can be seen, the mean is around $\hat{\rho}=$ 0.44 and the estimates do not average out. In order to test the significance of the correlation coefficient, one needs to know the distribution of $\hat{\rho}$. For some special cases it is possible to determine the distribution. But in general one will have to be satisfied with an approximation using a normal distribution, which is not unrealistic when the redundancy of the model is large. The correlation between $\mathrm{C} 1$ and $\mathrm{P} 2$ code observations for the 4000SSI turns out to be significant. This is verified when we compare the mean correlation coefficient with its precision, namely $\sigma_{\hat{\rho}}=$ 0.007 . This can also be simply resulted from the Chebyschev inequality even when one does not specify a distribution for $\hat{\rho}$.

To evaluate the satellite elevation dependence of the GPS observables, 3 satellites have been employed. Figure 3 shows the groupwise estimates of variance components using the full hour of the data (C1 code) of the satellites PRN 05, 29 and 09 . The variance components computed for satellite PRN 09, with the lowest elevation angle, are larger than those estimated for satellites PRN 05 and 29. Also, as the elevation angle decreases, a positive trend is observed (for the last groups, on average, the estimated variance components are larger than those

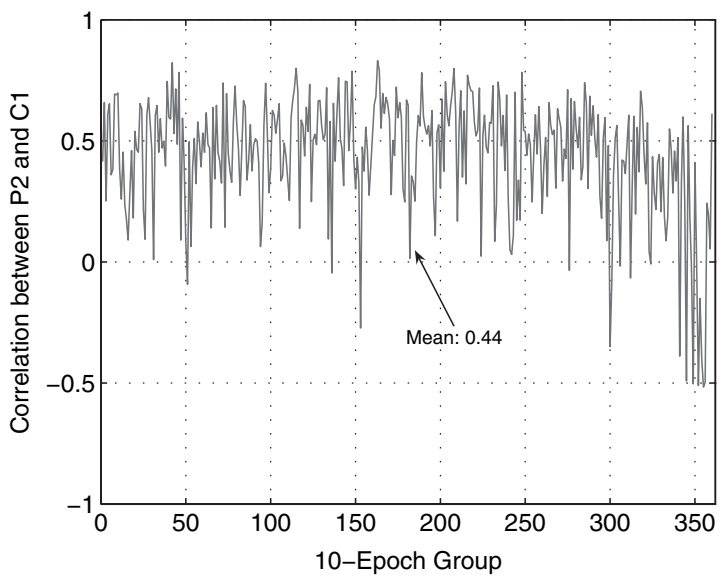

Fig. 2. Groupwise estimated correlation coefficient between $\mathrm{C} 1$ and P2 codes obtained from LS (co)variance estimates.

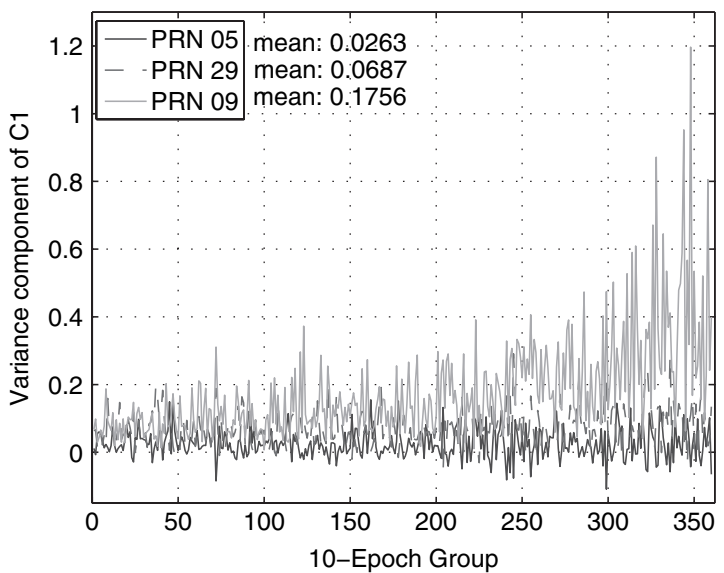

Fig. 3. Groupwise estimated variance components of $\mathrm{C} 1$ code estimated for different satellites. Factors are to be multiplied by $30^{2} \mathrm{~cm}^{2}$ for code observables.

of the first groups). Another point is that the variance components estimated for satellites PRN 05 and 29 are negatively correlated. This can also be obtained from the covariance matrix of the estimates $Q_{\hat{\sigma}}=$ $N^{-1}$. The correlation coefficients between satellites PRN 05 and 29 are $\hat{\rho}_{\phi}=-0.70, \hat{\rho}_{c 1}=-0.63$ and $\hat{\rho}_{p 2}=-0.67$, for phase, $\mathrm{C} 1$, and $\mathrm{P} 2$, respectively. This implies that the precision of the data of the satellites PRNs 29 and 05 is not much different. This makes sense since they both have high elevation angles. The numerical results indicate that the noise of satellites PRN 05-29-09 observations is about 0.2$0.2-0.8 \mathrm{~mm}, 5-8-13 \mathrm{~cm}$ and $4-12-33 \mathrm{~cm}$ for phase, $\mathrm{C} 1$, and $\mathrm{P} 2$, respectively. The precision of these estimates is at a few micrometre level, one millimetre level, and a few millimetre level, respectively.

\section{GPS Coordinate Time Series}

In this section we assess the noise characteristics in time series of daily position estimates for permanent GPS stations using LS-VCE. The precision of these estimates is often assessed by their repeatability defined by the mean squared error (MSE) of individual coordinate components (i.e. north, east, and up) about a linear trend. Except for a significant episodic deformation, such as large earthquakes, a linear trend can be a good representation of the (long term) deformation behavior. Therefore, the site velocities are usually determined by linear regression of individual coordinate components.

Previous work reveals the presence of white noise, flicker noise, and random walk noise in the GPS time series (see e.g. Langbein and Johnson, 1997, Zhang et al., 1997). If we now assume that the time series of GPS coordinates are composed of white 
noise with variance $\sigma_{w}^{2}$, flicker noise with variance $\sigma_{f}^{2}$, and random walk noise with variance $\sigma_{r w}^{2}$, the covariance matrix of the time series can be written as $Q_{y}=\sigma_{w}^{2} I+\sigma_{f}^{2} Q_{f}+\sigma_{r w}^{2} Q_{r w}$ where $I$ is the $m \times m$ identity matrix, and $Q_{f}$ and $Q_{r w}$ are the cofactor matrices relating to flicker noise and random walk noise, respectively. The structure of the matrix $Q_{y}$ is known (through $I, Q_{f}$, and $Q_{r w}$ ), but the contributions through $\sigma_{w}, \sigma_{f}$, and $\sigma_{r w}$ are unknown. The variance components $\sigma_{w}^{2}, \sigma_{f}^{2}$ and $\sigma_{r w}^{2}$ can now be estimated using the method of LS-VCE.

We analyze global time series of site positions which are supposed to have more noise than those from a regional solution. The daily GPS solutions of 5 stations, namely KOSG, WSRT, ONSA, GRAZ and ALGO, processed by the GPS analysis center at the Jet Propulsion Laboratory (JPL), are adopted. In geophysical studies, for most available time series, only one of $\sigma_{f}^{2}$ and $\sigma_{r w}^{2}$ is estimated along with $\sigma_{w}^{2}$ (Mao et al., 1999). One advantage of LS-VCE is the possibility of hypothesis testing with the stochastic model. Using the w-test statistic, we can in fact decide which noise component, in addition to white noise, is likely to be present in the time series; either flicker noise or random walk noise. Based on the results (not included here), a combination of white noise plus flicker noise turns out in general to best characterize the noise in all three position components (see Amiri-Simkooei et al., 2007).

Table 2 gives the white and flicker noise amplitudes for two stochastic models. We find in general that the horizontal components are less noisy than the vertical components by a factor of $2-4$. Compared to the white noise model only, the amplitude of white noise for the white noise plus flicker noise model is about $30 \%$ smaller. There exists significant flicker noise in the data (compare flicker noise amplitudes with their precision). We have obtained that the (formal) standard deviations of the site velocity for white noise plus flicker noise model compared to those for the pure white noise model are larger by factors of 10-15. Therefore, the simple pure white noise model gives too optimistic results for the site velocity uncertainty.

A significant and comparable amount of flicker noise (between sites) may reflect a common physical basis, such as seasonal atmospheric mass distribution, atmospheric noise, or second order ionospheric effects. Reduction in time-correlated noise from global solutions to regional solutions suggests that some of the noise is spatially correlated (Williams et al., 2004). An issue related to the noise in global time series is the impact of spatial correlation on the rate uncertainties.

Using the LS-VCE, we have estimated the spatial correlation, each time between two stations whereby we obtain one correlation coefficient for each noise component. Table 3 gives the numerical results. The table includes the spatial correlation coefficients of noise components (white and flicker noise). Both noise components seem to be spatially correlated to some extent. The spatial correlation of white noise (absolute values) is less than that of flicker noise, on average, by factors of $0.90,0.70$, and 0.65 for

Table 2. White noise and flicker noise amplitude estimates obtained by LS-VCE for north, east, and up components of site time series for two stochastic models (white noise only: WN, combination of white noise and flicker noise: WN+FN); $\sigma$ is standard deviation of estimator; N: north, E: east, and U: up component

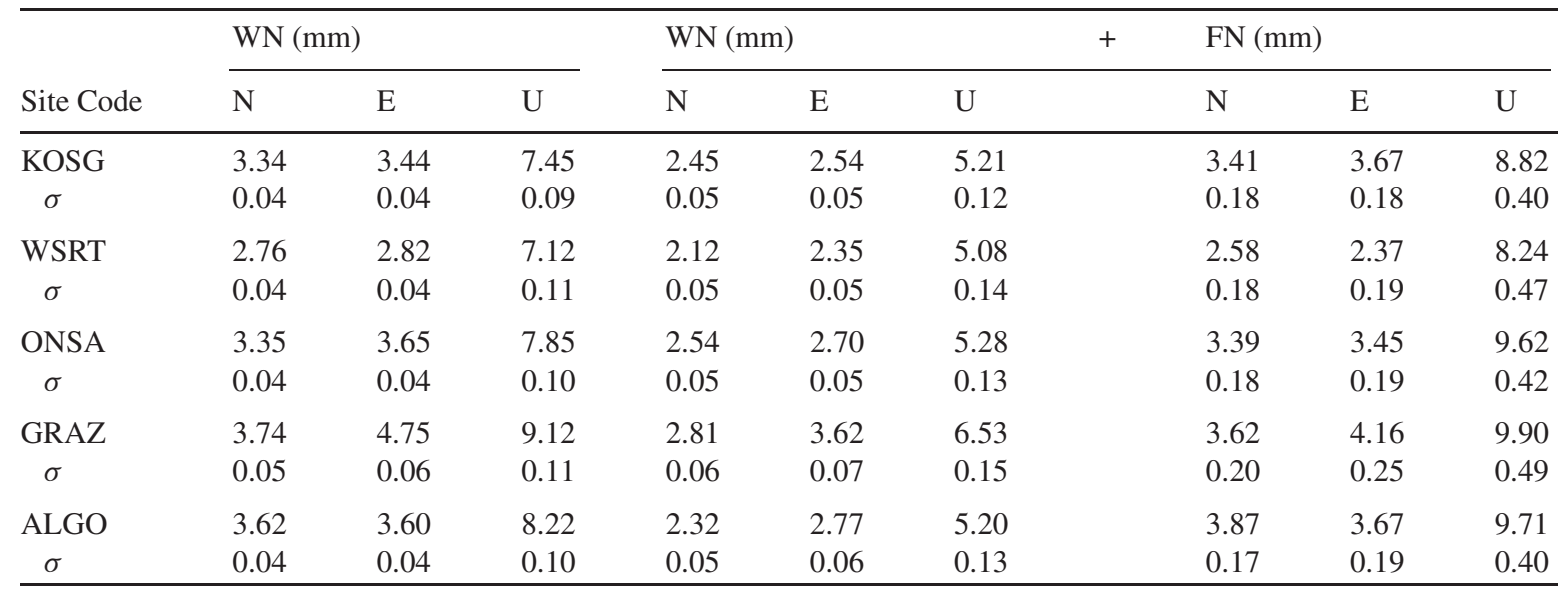


Table 3. Estimated spatial correlation coefficients of white noise (top) and flicker noise (bottom) components obtained from LS (co)variance estimates (using LS-VCE). Functional model consists of two time series, i.e. between corresponding $\mathrm{N}$ : north, E: east, or U: up components, and stochastic model includes four white and flicker noise variance components as well as two covariances of noise components between the two series. This table presents only correlation coefficients. Precision of estimators ranges from 0.01 to 0.10

\begin{tabular}{|c|c|c|c|c|c|}
\hline & & & \multicolumn{3}{|c|}{ Correlation coefficient } \\
\hline & \multicolumn{2}{|c|}{ Between Sites } & $\mathrm{N}$ & $\mathrm{E}$ & $\mathrm{U}$ \\
\hline & KOSG & WSRT & 0.85 & 0.61 & 0.70 \\
\hline & KOSG & ONSA & 0.69 & 0.46 & 0.52 \\
\hline & KOSG & GRAZ & 0.64 & 0.38 & 0.30 \\
\hline & KOSG & ALGO & 0.16 & -0.17 & -0.20 \\
\hline \multirow[t]{10}{*}{ WN } & WSRT & ONSA & 0.79 & 0.51 & 0.55 \\
\hline & WSRT & GRAZ & 0.76 & 0.48 & 0.49 \\
\hline & WSRT & ALGO & 0.30 & -0.13 & -0.09 \\
\hline & ONSA & GRAZ & 0.62 & 0.46 & 0.39 \\
\hline & ONSA & ALGO & 0.11 & -0.18 & -0.13 \\
\hline & GRAZ & ALGO & 0.14 & -0.12 & -0.09 \\
\hline & KOSG & WSRT & 0.92 & 0.89 & 0.88 \\
\hline & KOSG & ONSA & 0.80 & 0.69 & 0.72 \\
\hline & KOSG & GRAZ & 0.63 & 0.51 & 0.69 \\
\hline & KOSG & ALGO & 0.27 & -0.45 & -0.15 \\
\hline \multirow[t]{6}{*}{ FN } & WSRT & ONSA & 0.81 & 0.86 & 0.79 \\
\hline & WSRT & GRAZ & 0.75 & 0.50 & 0.83 \\
\hline & WSRT & ALGO & 0.20 & -0.07 & -0.28 \\
\hline & ONSA & GRAZ & 0.62 & 0.49 & 0.57 \\
\hline & ONSA & ALGO & 0.27 & -0.26 & -0.22 \\
\hline & GRAZ & ALGO & 0.32 & -0.35 & -0.29 \\
\hline
\end{tabular}

north, east, and up components, respectively. The maximum correlations for both noise components have been obtained between the nearest sites, i.e. between KOSG and WSRT (they are only $100 \mathrm{~km}$ apart). This confirms that the noise has a common physical basis. Over the largest station separation (between ALGO and other sites), the spatial correlation is the lowest for the north component. It becomes negative for the east and up components. These all together confirm that the site velocity between stations will be correlated as well. If one treats the time series individually, the correlation between time series should be added after into the covariance matrix of the site velocities.

\section{GPS Receiver Noise Characteristics}

We now consider a nonlinear variance component estimation problem. For this purpose we apply the LS-VCE to covariance functions. Using the Taylor series expansion, it is in principle possible to linearize the nonlinear stochastic model. When expanded into the Taylor series, the covariance matrix can be written as $Q_{y}=Q(\sigma) \approx Q_{0}+$ $\sum_{k=1}^{p} \sigma_{k} Q_{k}$. We can therefore apply the LS-VCE to estimate $\sigma$ (see Teunissen and Amiri-Simkooei, 2007). Since we linearize a nonlinear function, the solution should be sought through an iterative procedure. We can iterate until the estimated (co)variance components do not change by further iterations.

The goal, as an example, is to assess the noise characteristics of a GPS Trimble 4000SSI receiver (again data from the Delfland 99 campaign). Our point of departure here is the zero baseline time series. To obtain such baseline components, the single difference phase observation equation is employed. The data collected by the 4000SSI were static, but they were processed in kinematic mode (new unknown coordinates for every epoch). Baseline components and differential receiver clock biases along with double difference ambiguities were estimated by least-squares. We used the LAMBDA method to fix the ambiguities (see Teunissen, 1993). We therefore introduced the fixed ambiguities into the model (see Amiri-Simkooei and Tiberius, 2007, Tiberius and Kenselaar, 2000). We have now time series of zero baseline components with one second interval. This is considered as input for further assessment by LS-VCE. We will focus on time correlation.

In practice, covariance functions are formed by combining a small number of simple mathematically acceptable expressions or models. We will apply the method to the autoregressive noise (model I) and Gaussian noise (model II) models to the real data of the zero baseline test. The covariance functions related to these models are $Q_{1}(\tau)=$ $\sigma_{c}^{2} \exp (-\alpha \tau)$ and $Q_{2}(\tau)=\sigma_{c}^{2} \exp \left(-\alpha \tau^{2}\right)$, respectively. The parameters $\alpha$ and $\sigma_{c}^{2}$ are the time-scale (time-constant) and variance of the noise process, respectively. Both $\alpha$ and $\sigma_{c}^{2}$ are assumed to be unknown and should be estimated using the nonlinear LS-VCE. In each noise model, we will also include a white noise variance component, namely $\sigma_{w}^{2}$. Therefore, for each model, three unknown parameters are to be estimated; the variances $\sigma_{w}^{2}$ and $\sigma_{c}^{2}$ and the time-scale $\alpha$.

The numerical results are given in Table 4 . The results show that the 4000SSI is not free from time correlation. The variance components $\sigma_{w}^{2}$ and $\sigma_{c}^{2}$ of both stochastic models, when compared to their precision, are significant. This confirms that the time series contain white and colored noise components. The time-scale parameter $\alpha$ is on average 0.16 $\mathrm{sec}^{-1}$ and $0.04 \mathrm{sec}^{-2}$ for the autoregressive and 

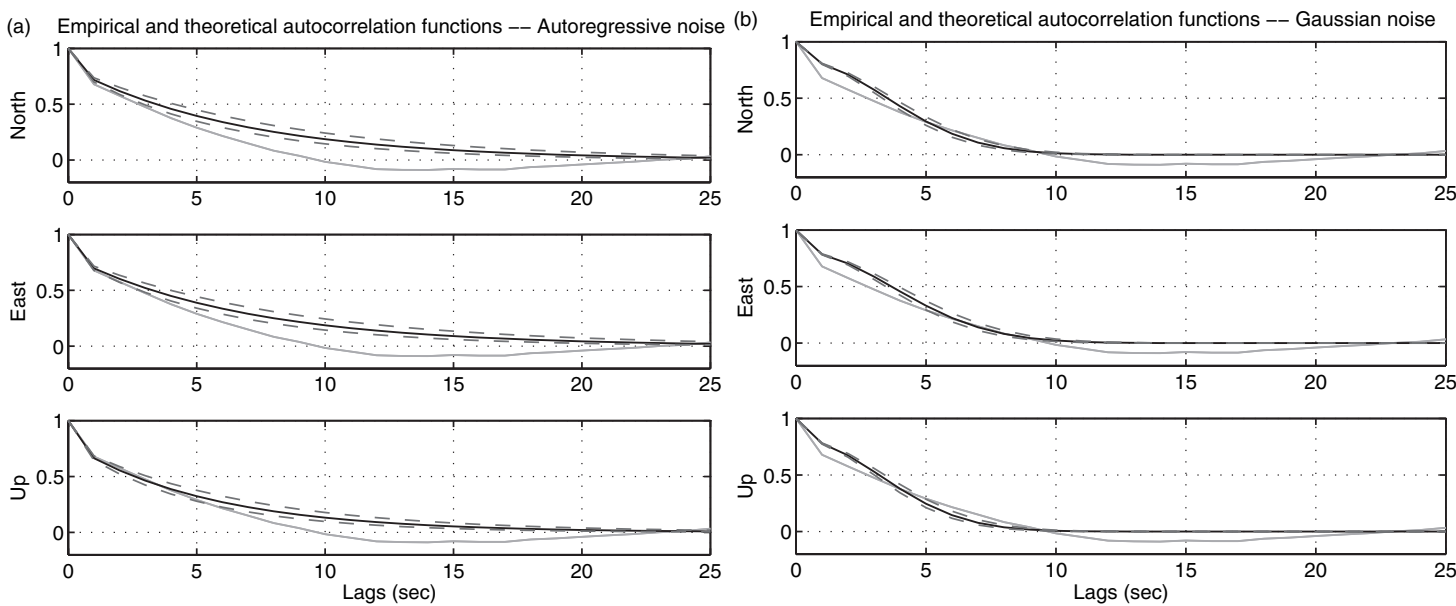

Fig. 4. Empirical (light line) and theoretical (black line) autocorrelation functions. Dashed lines show $95 \%$ confidence interval of theoretical autocorrelation function; white plus autoregressive noise model (a); white plus Gaussian noise model (b).

Gaussian noise models, respectively. This implies that there exist time correlation over about 10-20 seconds. One can also verify this by the empirical autocorrelation function which can simply be obtained from the least-squares residuals $\hat{e}_{i}$ as $\hat{\sigma}_{\tau}=$ $\left(\sum_{i=1}^{m-\tau} \hat{e}_{i} \hat{e}_{i+\tau}\right) /(m-\tau), \quad \tau=0, \ldots, m-1$ (see Teunissen and Amiri-Simkooei, 2007). On the other hand, we can define the theoretical autocorrelation function which is obtained from the LS estimates of $\hat{\sigma}_{w}, \hat{\sigma}_{c}$, and $\hat{\alpha}$. Figure 4 shows the empirical and theoretical autocorrelation functions which match each other well. Note that the empirical autocorrelation function resulted from the weighted LS-VCE when the weight matrix is taken as an identity matrix. The presence of a sharp bend in both the empirical and theoretical autocorrelation functions (at time lag of $\tau=1 \mathrm{sec}$ ) confirms that the noise of the time series

Table 4. Estimated variances $\sigma_{w}^{2}$ and $\sigma_{c}^{2}$ and time-scale $\alpha$ as well as their precision by LS-VCE; white plus autoregressive noise model (left); white plus Gaussian noise model (right)

\begin{tabular}{|c|c|c|c|c|c|}
\hline \multirow{2}{*}{\multicolumn{2}{|c|}{ Component }} & \multicolumn{2}{|c|}{ model I } & \multicolumn{2}{|c|}{ model II } \\
\hline & & $\hat{\sigma}$ & $\sigma_{\hat{\sigma}}$ & $\hat{\sigma}$ & $\sigma_{\hat{\sigma}}$ \\
\hline \multirow{3}{*}{$\mathrm{N}$} & $\sigma_{w}^{2}$ & 0.039 & 0.003 & 0.065 & 0.002 \\
\hline & $\alpha$ & 0.149 & 0.013 & 0.042 & 0.003 \\
\hline & $\sigma_{c}^{2}$ & 0.195 & 0.013 & 0.156 & 0.010 \\
\hline \multirow{3}{*}{ E } & $\sigma_{w}^{2}$ & 0.017 & 0.001 & 0.025 & 0.001 \\
\hline & $\alpha$ & 0.146 & 0.014 & 0.036 & 0.002 \\
\hline & $\sigma_{c}^{2}$ & 0.058 & 0.004 & 0.047 & 0.003 \\
\hline \multirow{3}{*}{$\mathrm{U}$} & $\sigma_{w}^{2}$ & 0.098 & 0.007 & 0.164 & 0.005 \\
\hline & $\alpha$ & 0.180 & 0.015 & 0.048 & 0.003 \\
\hline & $\sigma_{c}^{2}$ & 0.436 & 0.026 & 0.346 & 0.021 \\
\hline
\end{tabular}

is not all colored but partly white. This is also verified by the numerical results (see Table 4 ).

\section{Conclusions}

In this study we demonstrated that the general LSVCE can easily handle different models and serve different applications. We presented the results of three different GPS application examples of the method for which linear forms of the functional models were used. The goal was to assess the stochastic properties of GPS data. On the basis of the numerical results obtained, the following conclusions and remarks can be given:

- The LS-VCE model is a powerful method for estimation of the stochastic model parameters. It also provides the precision of the estimators. The method has several other attractive features (see Teunissen and Amiri-Simkooei, 2007). This method can therefore be introduced as a standard method for estimation (and also testing) of the (co)variance components in the stochastic model.

- In the GPS geometry-free model, LS-VCE was used to assess the noise characteristics of GPS observables. As expected, the variance of a GPS observable generally depends on the elevation of the satellite. Also, significant correlation can occur between different observation types, e.g. between the $\mathrm{C} 1$ and $\mathrm{P} 2$ codes. This is a good motivation to study the GPS stochastic model in more detail.

- The LS-VCE was applied to data of various permanent GPS tracking stations. It revealed that both white and flicker noise components are significant in GPS coordinate time series. In fact, 
ignoring the colored (flicker) noise, gives too optimistic results for the site velocity uncertainty. We also showed that both noise components are spatially correlated. The largest correlation coefficients were obtained between the nearest stations. This confirms that the noise has a common physical basis on the global time series.

- Finally, nonlinear LS-VCE was applied to assess the temporal correlation of GPS receivers based on covariance functions. The results showed that the 4000SSI GPS receiver is not free from time correlation. This was verified based on the empirical and theoretical autocorrelation functions.

\section{References}

Amiri-Simkooei, A. R. and Tiberius, C. C. J. M. (2007). Assessing receiver noise using GPS short baseline time series. GPS Solutions, 11(1), 21-35.

Amiri-Simkooei, A. R., Tiberius, C. C. J. M., and Teunissen, P. J. G. (2007). Assessment of noise in GPS coordinate time series: methodology and results, Journal of Geophysical Research, 112, B07413, doi:10.1029/2006JB004913.

Bischoff, W., Heck, B., Howind, J., and Teusch, A. (2005). A procedure for testing the assumption of homoscedasticity in least squares residuals: a case study of GPS carrier-phase observations. Journal of Geodesy, 78, 397-404.

Bischoff, W., Heck, B., Howind, J., and Teusch, A. (2006). A procedure for estimating the variance function of linear models and for checking the appropriateness of estimated variances: a case study of GPS carrier-phase observations. Journal of Geodesy, 79, 694-704.
Jonkman, N. F. (1998). Integer GPS ambiguity estimation without the receiver-satellite geometry. LGR-Series 18 , Geodetic Computing Centre, Delft.

Langbein, J. and Johnson, H. (1997). Correlated errors in geodetic time series: Implications for time-dependent deformation. Journal of Geophysical Research, 102(B1), 591-603.

Mao, A., Harrison, C. G. A., and Dixon, T. H. (1999). Noise in GPS coordinate time series. Journal of Geophysical Research, 104(B2), 2797-2816.

Teunissen, P. J. G. (1988). Towards a least-squares framework for adjusting and testing of both functional and stochastic model. Internal research memo, Geodetic Computing Centre, Delft. A reprint of original 1988 report is also available in 2004, No. 26, http://www.lr.tudelft.nl/mgp.

Teunissen, P. J. G. (1993). Least squares estimation of the integer GPS ambiguities. In Invited Lecture, Section IV, Theory and Methodology, IAG General Meeting, Beijing, China.

Teunissen, P. J. G. and Amiri-Simkooei, A. R. (2008). Least-squares variance component estimation. Journal of Geodesy (in press), doi 10.1007/s00190-007-0157-x.

Tiberius, C. C. J. M. and Kenselaar, F. (2000). Estimation of the stochastic model for GPS code and phase observables. Survey Review, 35(277), 441-454.

Williams, S. D. P., Bock, Y., Fang, P., Jamason, P., Nikolaidis, R. M., Prawirodirdjo, L., Miller, M., and Johnson, D. J. (2004). Error analysis of continuous GPS position time series. Journal of Geophysical Research, 109.

Zhang, J., Bock, Y., Johnson, H., Fang, P., Williams, S., Genrich, J., Wdowinski, S., and Behr, J. (1997). Southern California Permanent GPS Geodetic Array: Error analysis of daily position estimates and site velocities. Journal of Geophysical Research, 102, 18035-18055. 